\title{
Remarques sur la taxonomie infraspécifique de Sergentomyia minuta, Sergentomyia africana et Sergeniomyia antennata
}

\author{
par J.-A. RIOUX, H. CROSET, N. LEGER et M. MAISTRE \\ Laboratoire d'Ecologie médicale et de Pathologie parasitaire, \\ Faculté de Médecine, rue Auguste-Broussonnet, F 34000 Montpellier \\ et Laboratoire de Parasitologie, Faculté de Pharmacie, \\ 4, avenue de l'Observatoire, F 75006 Paris
}

\section{Résumé.}

A l'aide de trois exemples, les auteurs discutent de la valeur systématique de l'armature cibariale dans le genre Sergentomyia. Ainsi, la distribution des dents ne permet pas de distinguer les sous-espèces, voire les espèces, décrites dans les complexes antennata et africana. Bien plus, dans le complexe minuta, ce caractère se présente comme un «cline» typique le long de la côte méditerranéenne de l'Espagne.

\section{Summary.}

Comments on the infraspecific taxonomy of Sergentomyia minuta (Rondani, 1843), S. africana (Newstead, 1912) and S. antennala (Newstead, 1912).

The authors discuss the systematic value of the cibarial armature of three members of the genus Sergentomyia. Subspecies could not be differentiated by the number of teeth, nor could members of the antennata and africana complexes. Moreover, from this character, the minuta complex along the mediterranean coast of Spain appears to represent a typical «cline». 
Certes, la systématique des Diptères Phlebotominae atteint actuellement un seuil d'analyse typologique satisfaisant. Pour les régions paléarctique et, pro parte, éthiopienne, l'effort de prospection réalisé depuis le début du siècie permet de disposer d'études monographiques de qualité. Toutefois, les principes taxonomiques qui soustendent nombre de ces travaux, se réfèrent à des concepts fixistes d'où sont exclues les notions de modalités et de niveaux de spéciation.

Une telle attitude amène à négliger l'une des données fondamentales sur laquelle s'appuie aujourd'hui toute étude biosystématique : la population naturelle, unité reproductive élémentaire, phénotypiquement et géographiquement définie. L'utilisation de critères d'identification insuffisamment éprouvés, et partant fallacieux, en sont malheureusement, la conséquence involontaire.

A la décharge des systématiciens de ce groupe, il faut toutefois noter les difficultés réelles des approches génétique et écologique: les récoltes massives sont exceptionnelles, les larves rarement dépistées in natura et les élevages en continu, hasardeux. Pour l'ensemble de ces raisons, les Phlébotomes ne bénéficient pas encore des techniques morphométriques, chromosomiques ou mixiologiques couramment utilisées à l'endroit d'autres familles de Vecteurs hématophages, tels que les Culicidae et, à un degré moindre, les Simulidae.

Il nous a donc paru utile de rouvrir le dossier des taxons subspécifiques des Phlebotominae sur la base de données quantitatives et ce, dans une acceptation nouvelle, directement issue des concepts «synthétiques» actuels. Sans prétendre proposer et moins encore, imposer une véritable stratégie, nous voudrions simplement présenter le problème des critères biométriques à l'aide de trois exemples tirés d'une récente prospection épidémiologique en Espagne et au Maroc. Ce faisant, nous souhaitons attirer l'attention sur l'intérêt d'une analyse statistique des critères systématiques et sur l'importance du rôle des facteurs géographiques dans la diversification infraspécifique.

\section{Sergentomyia (Sergentomyia) minuta (Rondani, 1843)}

Depuis les travaux de L. Parrot (1942) et O. Théodor (1958), l'espèce circuméditerranéenne Sergentomyia minuta est considérée comme formée de deux sous-espèces (fig. 1), l'une (ssp. minuta), de répartition européenne, à cibarium paucidenté (moyenne des dents: 40), l'autre (ssp. parroti) de répartition nord africaine, à denture cibariale forte (moyenne des dents: 70).

L'étude de plusieurs séries d'échantillons, capturés en 1972 et 1973 , le long d'un transect Espagne-Maroc, nous permet aujourd'hui d'infirmer cette conception. A partir de Valence apparaissent en effet, des formes se rapprochant du type «parroti». Le nombre de dents augmente vers le Sud, jusqu'à atteindre, de manière assez pro'gressive, sa valeur maximale aux environs de Gibraltar. Ce chiffre semble se maintenir par la suite, sur l'ensemble du Maroc (fig. 2). 
Dans la partie occidentale de son aire, en particulier sur la côte méditerranéenne de l'Espagne, Sergentomyia minuta se comporte donc comme un «cline». S'agit-il d'un cline vrai, c'est-à-dire de la modification progressive d'une même entité taxonomique soumise à un gradient écologique régulier, ou d'un cas d'hybridation allopatrique correspondant à l'intégration de deux sous-espèces géographiques, il est pour l'instant difficile de se prononcer. Or, la nomenclature trinominale ne peut être appliquée que dans la deuxième éventualité et encore, en dehors de la péninsule ibérique, s'il se confirme qu'effectivement le phénomène clinal s'étend des Pyrénées au détroit de Gibraltar.

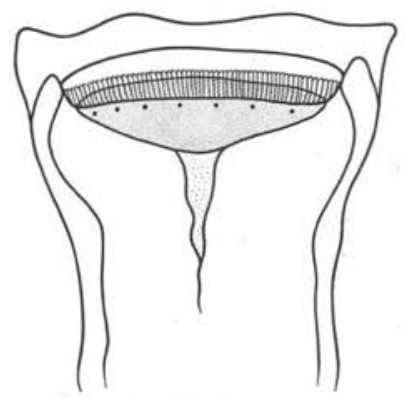

PE 41

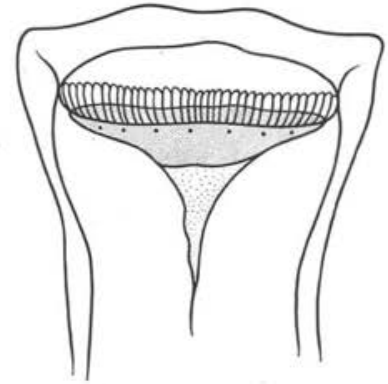

TC 435

FIG. 1. - Les deux «sous-espèces» de Sergentomyia minuta (Rondani 1843) : la «sous-espèce » minuta, à pharynx paucidenté (d'après un exemplaire récolté en Corse, T.C. 435) et la «sousespèce » parroti à pharynx fortement armé (d'après un exemplaire récolté en Espagne aux environs d'Algésiras, PE41).

Sergentomyia (Parrotomyia) africana (Newstead, 1912)

A ce jour, le «complexe»Sergentomyia africana réunit-un nombre important d'espèces, sous-espèces et «variétés ». Dans une monographie récente, E. Abonnenc et P. Yvore ont remạrquablement analysé ce groupe en montrant les difficultés de procéder au regroupement des formes affines. Dans la région méditerranéenne trois sous-espèces ont été individualisées : Sergentomyia africana asiatica (Théodor, 1939), S. africana cherifiana (Ristorcelli, 1939) et S. africana eremitis (Parrot et de Jolinière, 1945) dont la distinction repose sur le nombre de dents du cibarium et le type de formule palpale.

Au cours d'une mission réalisée en 1973 dans le sud marocain, nous avons pu capturer un lot de 1361 exemplaires $\left(\begin{array}{lll}1 & 196 & \text { et } \\ 165 & \delta^{*}\end{array}\right)$ de $S$. africana dans la palme- 
Sergentomyia minuta (RONDANI, 1843)

ARMATURE CIBARIALE

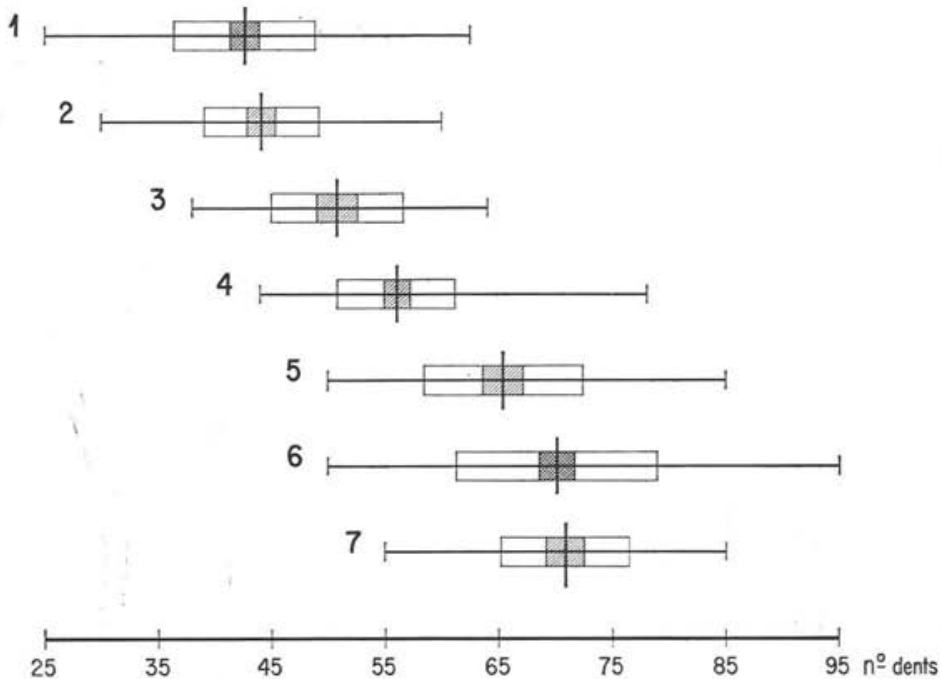

FIg. 2. - Le cline Sergentomyia minuta (Rondani, 1843).

1. Sud de la France (synthétique) $: n: 136, m: 42,6, \sigma: 6,29$.

2. Chuisa - Vallée de Cruzzini (Corse), 27.VII.72, TC 435; $n: 60, m: 44,3, \sigma: 5,04$.

3. Chilches N. 340 (Castellon), 29.VIII.72, PE $2 ; n: 40, m: 50,75, \sigma: 5,6010$.

4. Puerto Lumbreras (Murcia), 22.VIII.73, PE $30 ; n: 109, m: 56,05, \sigma: 5,2979$.

5. Boujad (Maroc), 30.VIII.73, TM $122 ; n: 60, m: 65,5, \sigma: 6,96$.

6. Tunisie du Nord (synthétique) $: n: 136, m: 70,2, \sigma: 6,96$.

7. Algésiras (Espagne), 24.VIII.73, 25.VIII.73, PE 41; $n: 49, m: 70,8, \sigma: 5,64$.

Trait vertical: moyenne de l'échantillon; rectangle hachuré: intervalle de confiance de la moyenne; rectangle blanc : écart-type de l'échantillon; ligne horizontale: extrêmes observés.

raie de $\mathrm{Agdz}$ (technique des pièges adhésifs : 160 pièges tendus du 12.9 .73 au 16.9.73). L'étude des critères taxonomiques traditionnels nous ont amenés à constater que :

- les dimensions des articles des palpes ne permettaient pas de rattacher les exemplaires marocains à l'une ou l'autre des trois espèces citées ;

- les valeurs obtenues par le dénombrement des dents cibariales (fig. 3) couvraient les données considérées jusqu'à présent, comme caractéristiques des sous-espèces asiatica et eremitis. 
Rappelons que E. Abonnenc et P. Yvore (loc. cit.) avaient déjà considéré $S$. africana cherifiana comme synonyme de S. africana asiatica. Selon eux, S. africana eremitis devrait conserver sa place systématique en raison du faible nombre de dents cibariales (48 contre 58 à 65 ) et de l'absence d'encoche sur le bord antérieur de l'aire pigmentée. Elargissant la conception uniciste de ces auteurs, il nous paraît actuellement rationnel de réunir en un seul taxon les «formes » paléarctiques, c'est-à-dire nord-africaines et sahariennes, du complexe «africana» et, par conséquent, inutile d'utiliser à leur endroit, la nomenclature trinominale.

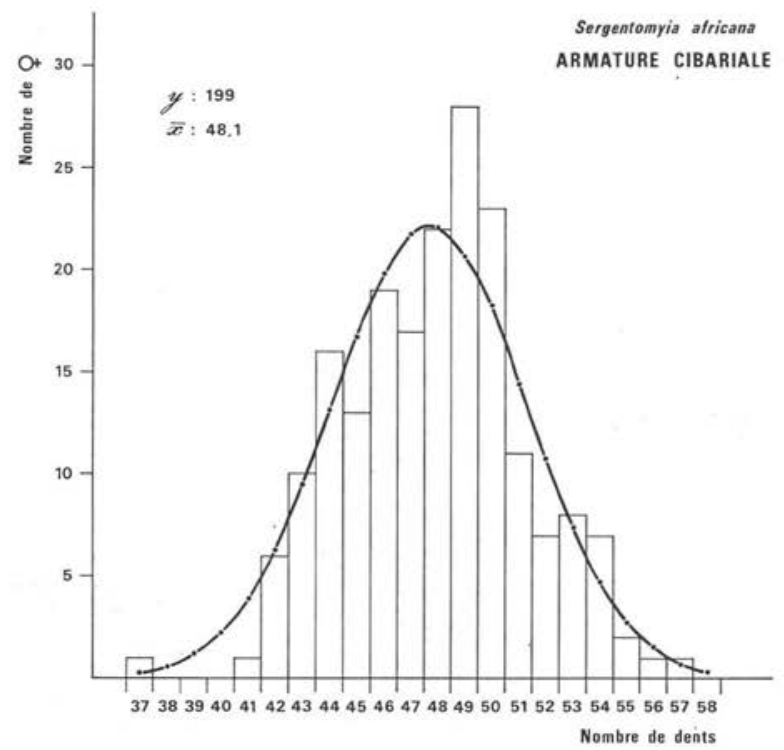

FIG. 3. - Sergentomyia africana (Newstead, 1912). Distribution des dents cibariales dans une population capturée à Agz (TM 198).

Sergentomyia (Sergentomyia) antennata (Newstead, 1912)

Sur la base d'une étude biométrique de 704 exemplaires $\$$ marocains capturés en 1972 (N. Léger et coll., 1974), nous sommes parvenus à une conclusion identique pour Sergentomyia antennata: l'armature cibariale, en particulier, présente tous les intermédiaires entre les formes cincta et antennata (fig. 4). 


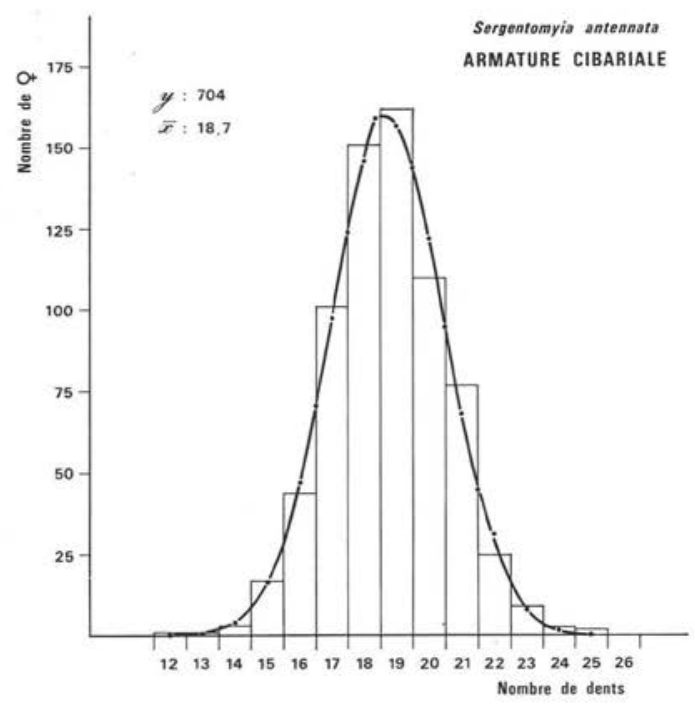

FIG. 4. - Sergentomyia antennata (Newstead, 1912). Distribution du nombre de dents du cibarium dans les populations du Sud marocain. Les «espèces » Sergentomyia antennata (Newstead, 1912) et Sergentomyia cincta (Parrot et Martin, 1944) ne peuvent être distinguées par l'armature cibariale).

En définitive, il paraît urgent de reconsidérer la distribution des dents cibariales en tant que critère systématique. Dans certains cas (Sergentomyia africana, Sergentomyia antennata), la grande dispersion statistique enlève à ce caractère, toute valeur discriminante au niveau infraspécifique voire spécifique. Dans d'autres (Sergentomyia minuta), la sensibilité aux facteurs climatiques (cline), tout en présentant un intérêt conceptuel non négligeable, aboutit en pratique aux mêmes inconvénients.

\section{Bibliographie}

Abonnenc (E.), 1972. - Les Phlébotomes de la région éthiopienne (Diptera, Psychodidae). Mém. O.R.S.T.O.M., 55, 289 p.

AbonNenc (E.) et Yvore (P.), 1969. - Phlébotomes du complexe «africanus 》 (Diptera, Psychodidae). Cah. O.R.S.T.O.M., sér. Ent. méd. Parasitol., 7, 181-208.

Adler (S.) et Theodor (O.), 1926. - On the minutus group of the genus Phlebotomus in Palestine. Bull. ent. Res., 16, 399-405.

Bailly-Choumara (H.), Abonnenc (E.) et Pastre (J.), 1971. - Contribution à l'étude des Phlébotomes du Maroc (Diptera, Psychodidae). Données faunistiques et écologiques. Cah. O.R.S.T.O.M., sér. Ent. méd. Parasitol., 9, 431-460. 
Léger (N.), Rioux (J.-A.), Croset (H.), Cadi Soussi (M.) et Ben Mansour (N.), 1974. Le «complexe» Sergentomyia (Sergentomyia) antennata (Newstead, 1912). Ann. Parasit. hum. comp., 49, 577-591.

MAYR (E.), 1970. - Population, Species and Evolution. Harward University Press Publ., Cambridge, U.S.A., 496 p.

NAJERA (L.), 1943. - Nuevos datos sobre la distribucion geografica de los «Phlebotomus » en Espagne. Graellsia, 1, 1-12.

Newstead (R.), 1912. - Notes on Phlebotomus with descriptions of New species. Part I. Bull. ent. Res., 3, 361-367.

Parrot (R.), 1929. - Notes sur les Phlébotomes. III. 2. Morphologie et répartition géographique de Phlebotomus parroti Adler et Theodor. Arch. Inst. Pasteur, Algérie, 7, 306-307.

Rioux (J.-A.), Golvan (Y.-J.), Croset (H.), Tour (S.), Houin (R.), Abonnenc (E.), Petitdidier (M.), Vollhardt (Y.), Dédet (J.-P.), Albaret (J.-L.), Lanotte (G.) et Quilici (M.), 1969. - Epidémiologie des Leishmanioses dans le sud de la France. Monographie I.N.S.E.R.M., 223 p.

SACCA (G.), 1947. - Revisione dei Phlebotomus della collezione Rondani ; un punto fermo sulla questione del P. minutus. Rend. Ist. Sup. San., 10, 925-934.

Theodor (O.), 1958. - Psychodidae - Phlebotominae, in: Die Fliegen der Palearktischen Region, E. Schweizerbart, édit., Stuttgart, $55 \mathrm{p}$. 